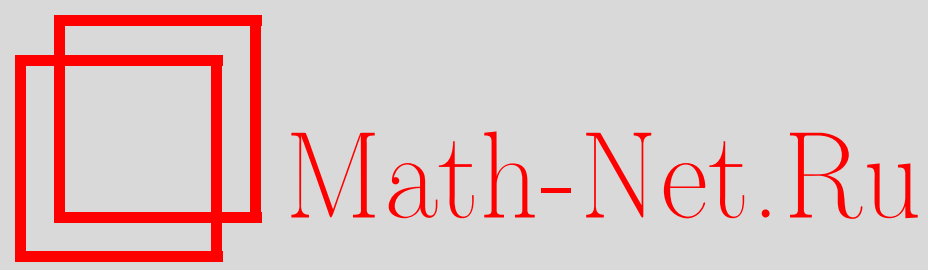

Я. Б. Воробец, Бильярды в рациональных многоугольниках: периодические траектории, симметрии, $d-$ устойчивость, Матем. заметки, 1997, том 62, выпуск 1, 6675

DOI: https://doi.org/10.4213/mzm1588

Использование Общероссийского математического портала Math-Net.Ru подразумевает, что вы прочитали и согласны с пользовательским соглашением http://www.mathnet.ru/rus/agreement

Параметры загрузки:

IP : 54.198 .64 .247

26 апреля 2023 г., $18: 37: 27$

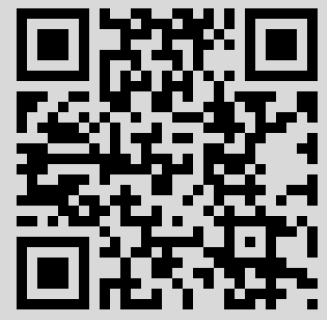




\title{
БИЛЬЯРДЫ В РАЦИОНАЛЬНЫХ МНОГОУГОЛЬНИКАХ: ПЕРИОДИЧЕСКИЕ ТРАЕКТОРИИ, СИММЕТРИИ, $d$-УСТОЙЧИВОСТЬ
}

\author{
Я.Б. Воробец
}

В статье рассматриваются периодические траектории бильярдов в рациональных многоугольниках, удовлетворяющих альтернативе Вича, в том числе в прямоугольных треугольниках с одним острым углом вида $\pi / n$, где $n$ - целое.

Среди затрагиваемых вопросов: симметричность периодических траекторий, асимптотика числа траекторий, не превышающих заданной длины, устойчивость периодических бильярдных траекторий к малым деформациям многоугольника.

Библиограффия: 5 названий.

Введение. Бильярдны й поток в многоугольнике $Q$ описывает прямолинейное движение точки внутри $Q$ с единичной скоростью, с отражением от границы многоугольника по закону “угол падения равен углу отражения". Фазовьм пространством потока является прямое произведение $Q$ на окружность “единичных скоростей” $S^{1}$, с необходимыми отождествлениями на его гранище, вытекающими из закона отражения. Проекция фазовой кривой на многоугольник назьвается бильярдной траекторией. Бильярдная траектория, попадаюшая в вершину $Q$, назьвается особой, ее дальнейшее продолжение, вообще говоря, не определено. Траектория, выходящая из одной вершины многоугольника и заканчивающаяся в другой вершине (т.е. двусторонне особая), назьвается обобщенной диагональю.

В дальнейшем предполагается, что многоугольник $Q$ рационален, т.е. угол между любыми двумя его сторонами рационально соизмерим с $\pi$. При этом все звенья фиксированной бильярдной траектории параллельны лишш конечному числу направлений. В результате фазовое пространство $Q \times S^{1}$ разбивается на инвариантные поверхности, “склеенные" из конечного числа копий многоугольника $Q$ (вида $Q \times\{\bar{v}\}, \bar{v} \in S^{1}$ ). Все эти поверхности, кроме двух исключительных, гомеоморфны одной и той же связной компактной ориентируемой поверхности $M$ и согласованным образом задают на ней плоскую структуру, т.е. риманову метрику нулевой кривизны с конечным числом конических особых точек, угол в каждой особой точке кратен $2 \pi$ (подробнее см., например, в [1]).

О бильярдных потоках в многоугольниках общего вида известно немного. В то время как бильярды в рациональных многоугольниках изучены достаточно хорошо.

Работа выполнена при финансовой поддержке Российского фонда фундаментальных исследований, грант № 96-01-00713, и Международного научного фонда, грант M1E300. 
В этой статье рассматривается ряд вопросов о периодических бильярдных траекториях в рациональном многоугольнике, для которого соответствующая поверхность с плоской структурой имеет богатую группу аффинных симметрий (подробнее см. в п. 1). Такие многоугольники рассматривались в работе [1]. Их важнейшим свойством является обнаруженная Вичем [2] альтернатива: каждая неособая траектория бильярда либо периодична, либо равнораспределена в многоугольнике.

Среди рассматриваемых ниже вопросов - симметричность периодических траекторий, асимптотика числа периодических траекторий длины не больше $R$, деформационная устойчивость периодических траекторий в многоугольнике (т.е. устойчивость к малым деформациям многоугольника). Один из результатов статьи (о деформационной неустойчивости всех периодических бильярдных траекторий в прямоугольном треугольнике с острьм углом вида $\pi /(2 n)$, где $n$ - целое) был анонсирован в [3].

Автор благодарен А. М. Стёпину за полезные замечания и обсуждения.

1. Аффинные симметрии. Уточним определение плоской структуры, данное во введении. Итак, мы имеем на поверхности $M$ риманову метрику нулевой кривизны с конечньм числом особых точек, в каждой из которых - коническая особенность с углом $2 \pi m$, где $m$ - натуральное число (кратность особой точки). При этом у произвольной неособой точки $x \in M$ найдется окрестность $U_{x}$, изометричная некоторой области в $\mathbb{R}^{2}$. Каждое изометрическое вложение окрестности в $\mathbb{R}^{2}$ задает в $U_{x}$ локальную систему декартовых координат. Системы координат, задаваемые разньми вложениями, отличаются на поворот, сдвиг и, возможно, отражение. Ввиду того, что грушпа голономии рассматриваемой римановой метрики на $M$ тривиальна, можно выбрать локальные декартовы координаты в окрестности каждой неособой точки так, чтобы в пересечении любых двух окрестностей соответствующие системы координат отличались только сдвигом. Такой набор локальных систем координат назовем декартовыми координатами на поверхности $M$. В дальнейшем под плоской структурой подразумевается риманова метрика указанного выше вида, снабженная декартовьми координатами.

Каждую кривую на поверхности $M$ посредством декартовых координат можно перенести в $\mathbb{R}^{2}$, получив ее развертку - плоскую кривую, определенную однозначно с точностью до всевозможных сдвигов в $\mathbb{R}^{2}$. Далее будут рассматриваться периодические геодезические на $M$ и седлосвязки - геодезические отрезки, соединяющие особые точки. Их развертки являются отрезками.

ОПРЕДЕЛЕНИЕ 1.1. Аффинной симметрией плоской структуры $\omega$ на поверхности $M$ назьвается гомеоморфизм поверхности $M$ на себя, которьй переводит особые точки плоской структуры в особые, а в окрестности каждой неособой точки является аффинным отображением в локальных декартовых координатах, соответствующих $\omega$.

Для любой аффинной симметрии однозначно определена ее линейная (однородная) часть - некоторая матрица размера $2 \times 2$ с определителем \pm 1 . Стабилизатором $Г(\omega)$ плоской структуры $\omega$ называется групша, состоящая из линейных частей аффинных симметрий, сохраняющих ориентацию поверхности. Стабилизатор является дискретной подгруппой в $\mathrm{SL}(2, \mathbb{R})$ (см. [1], [2]). Принято говорить, что плоская структура $\omega$ имеет большое число аффинных симметрий, если $\Gamma(\omega)$ - решетка в групше $\mathrm{SL}(2, \mathbb{R})$, т.е. однородное пространство $\mathrm{SL}(2, \mathbb{R}) / \Gamma(\omega)$ имеет конечный объем. 
Пусть известно, что $\Gamma(\omega)$ содержит некоторую решетку $\Gamma$. Поскольку стабилизатор - дискретная подгруппа в $\mathrm{SL}(2, \mathbb{R})$, он также является решеткой, и $\Gamma$ - подгруппа конечного индекса в $\Gamma(\omega)$. Ниже будет сформулировано достаточное условие для того, чтобы $\Gamma$ совпадала со всем стабилизатором $\Gamma(\omega)$. Предварительно введем некоторые определения. Пусть $\bar{v} \in S^{1}$ - вектор, параллельньй седлосвязке плоской структуры $\omega$. Согласно альтернативе Вича (см. [1], [2]) все неособые геодезические, параллельные $\bar{v}$, периодичны и образуют конечное число пучков, разделяемых седлосвязками. $l$-последовательностью для направления $\bar{v}$ назовем набор длин указанных пучков, $r$-последовательностью для $\bar{v}$ назовем набор отношений длины к ширине для тех же пучков. При этом все элементы $r$-последовательности соизмеримы друг с другом (см. [1]).

Далее, напомним, что важной характеристикой произвольной решетки $\Gamma \in \mathrm{SL}(2, \mathbb{R})$ является число ее параболических вериин (каспов), т.е. наибольшее число вершин на абсолюте у фундаментального многоугольника для действия Г на плоскости Лобачевского (см. [4]), попарно не отождествляемых этим действием.

ПРЕДЛОЖЕНИЕ 1.1. Пусть стабилизатор $Г(\omega)$ плоской структуры $\omega$ содержит решетку $\Gamma$, имеющую $k$ параболических вершин. Предположим, что нашлось $в$ точности $k$ направлений $\bar{v}_{1}, \ldots, \bar{v}_{k} \in S^{1}$, параллельных седлосвязкам структуры $\omega$, таких, что для каждой пары направлений $\bar{v}_{i} u \bar{v}_{j}(i \neq j)$ соответствующие им $r$-последовательности или l-последовательности не совпадают с точностью до перестановки членов и умножения их всех на одно и то же число. Тогда $\Gamma(\omega)=\Gamma$, если всякий әлемент $a \in \Gamma(\omega)$ такой, что а $\bar{v}_{1}= \pm \bar{v}_{1}$, содержится $в$ Г. Последнее условие имеет место, например, при одновременном выполнении следующих требований:

i) $\Gamma$ coдержит оператор $b$ такой, что $b \bar{v}_{1}=\bar{v}_{1}, b \bar{u}_{1}=\bar{u}_{1}+r \bar{v}_{1}$, где $\bar{u}_{1}-e \partial u$ ничный вектор, ортогональный $\bar{v}_{1}, r$ - наименьшее положительное число, которое делится нацело на каждый әлемент $r$-последовательности для направления $\bar{v}_{1}$;

ii) для некоторого $l \neq 0$ аффиннымми симметриями $\omega$ с линейными частями из Г можно реализовать любую перестановку на (непустом) множестве седлосвязок с разверткой $l \bar{v}_{1}$;

iii) хотя бы одна седлосвязка с разверткой $l \bar{v}_{1}$ принадлежит общей части границы двух неконгруәнтных периодических пучков, или жсе все такие седлосвязки переводятся в себя некоторой аффинной симметрией с линейной частью $-1,-1 \in \Gamma$;

iv) все седлосвязки, параллельные $\bar{v}_{1}$, входят в мноэсество $U$, полученное из поверхности $M$ после удаления из нее тех пучков периодических геодезических, параллельных $\bar{v}_{1}$, компонента гранищы которых содержит две седлосвязки равной длины, а также удаления особых точек; вдобавок, каждая компонента связности множества $U$ содержит седлосвязку с разверткой $l \bar{v}_{1}$.

ДокАЗАТЕЛЬСтво. Начнем со следующего замечания. Пусть $\bar{v}-$ вектор, параллельный седлосвязке структуры $\omega, \gamma$ - элемент $\Gamma(\omega)$. Тогда пучки периодических геодезических, параллельные $\bar{v}$, аффинной симметрией с линейной частью $\gamma$ переводятся в пучки периодических геодезических, параллельные $\gamma \bar{v}$. Отсюда следует, что $l$-последо- 
вательности для направлений $\bar{v}$ и $\gamma \bar{v}$ совпадают с точностью до перестановки членов и умножения их всех на одно и то же число. Тем же свойством обладают и $r$-последовательности для $\bar{v}$ и $\gamma \bar{v}$.

Поскольку $\Gamma$ - решетка в $\mathrm{SL}(2, \mathbb{R})$ с $k$ параболическими вершинами, можно выбрать $k$ ненулевых векторов $\bar{e}_{1}, \ldots, \bar{e}_{k}$ так, что любой вектор, который переходит в себя под действием нетождественного элемента решетки, некоторым элементом $Г$ переводится в вектор, коллинеарный одному из выбранных (см. [1, п. 3.4]). Согласно альтернативе Вича $[1$, теорема 3.4$]$ для вектора $\bar{v}$, параллельного седлосвязке, найдется элемент $a \in \Gamma(\omega), a \neq 1$, такой, что $a \bar{v}=\bar{v}$. Группа $\Gamma$ имеет конечньй индекс в $\Gamma(\omega)$, поэтому среди смежных классов $\Gamma, a \Gamma, a^{2} \Gamma, \ldots$ только конечное число различных. Тогда $a^{n} \in \Gamma$ для некоторого целого $n>0$. Имеем $a^{n} \bar{v}=\bar{v}, a^{n} \neq 1$, и, следовательно, вектор $\bar{v}$ оператором из Г переводится в вектор, коллинеарный некоторому $\bar{e}_{s}$. Ввиду приведенного выше свойства $l$ - и $r$-последовательностей векторы $\bar{v}_{i}$ и $\bar{v}_{j}(i \neq j)$ не могут переводиться в векторы, коллинеарные одному и тому же $\bar{e}_{s}$. Отсюда следует, что в качестве $\bar{e}_{1}, \ldots, \bar{e}_{k}$ можно взять векторы $\bar{v}_{1}, \ldots, \bar{v}_{k}$.

Пусть $\varphi$ - произвольная аффинная симметрия плоской структуры $\omega, a-$ ее линейная часть. Как показано выше, вектор $(\gamma a) \bar{v}_{1}$ при некотором $\gamma \in \Gamma$ коллинеарен некоторому $\bar{v}_{i}$. Из условия предложения следует, с учетом свойств $l$ - и $r$-последовательностей, что $\bar{v}_{i}=\bar{v}_{1}$, т.е. $(\gamma a) \bar{v}_{1}=\lambda \bar{v}_{1}$. Оператор $\gamma a$ переводит развертку седлосвязки в развертку седлосвязки, а так как седлосвязок, параллельных $\bar{v}_{1}$, конечное число, имеем $\lambda= \pm 1$. Поскольку $\gamma a \notin \Gamma$, если $a \notin \Gamma$, утверждение 1) доказано.

Перейдем к утверждению 2). Пусть $\varphi$ - аффинная симметрия с линейной частью $a$, $a \bar{v}_{1}= \pm \bar{v}_{1}$. Докажем, что $a \in \Gamma$. Ввиду условия іi) найдется аффинная симметрия $\varphi_{1}$ с линейной частью $a_{1} \in \Gamma$ такая, что $\varphi_{1} \varphi$ переводит в себя каждую седлосвязку с разверткой $l \bar{v}_{1}$. При этом $\left(a_{1} a\right) \bar{v}_{1}= \pm \bar{v}_{1}$. Более того, симметрию $\varphi_{1}$ можно выбрать так, чтобы $\left(a_{1} a\right) \bar{v}_{1}=\bar{v}_{1}$. Действительно, если $\left(a_{1} a\right) \bar{v}_{1}=-\bar{v}_{1}$, то любые два пучка периодических геодезических, имеющих на границе общую седлосвязку с разверткой $l \bar{v}_{1}$, переходят друг в друга при аффинной симметрии $\varphi_{1} \varphi$, что влечет конгруэнтность этих пучков. В этом случае можно “подправить" симметрию $\varphi_{1}$, воспользовавшись условиem iii).

Теперь рассмотрим произвольньй пучок периодических траекторий, параллельных $\bar{v}_{1}$. Если на его границе есть седлосвязка, неподвижная относительно симметрии $\varphi_{1} \varphi$, то таковыми будут все седлосвязки, ограничивающие пучок с той же стороны. А если среди седлосвязок, ограничивающих пучок с другой стороны, нет равных по длине, $\varphi_{1} \varphi$ оставляет на месте и их. Отсюда с учетом условия iv) и того, что $\varphi_{1} \varphi$ оставляет неподвижными седлосвязки с разверткой $l \bar{v}_{1}$, получаем, что $\varphi_{1} \varphi$ оставляет неподвижными вообще все седлосвязки, параллельные $\bar{v}_{1}$. В этом случае симметрия $\varphi_{1} \varphi$ переводит в себя каждьй пучок геодезических, параллельных $\bar{v}_{1}$, поэтому ее линейная часть есть степень оператора $b$ из условия i), т.е. принадлежит Г. Тогда и $a \in \Gamma$. Предложение доказано.

Ниже будут рассматриваться плоские структуры, каноническим образом построенные по рациональным треугольникам с двумя углами вида $\pi / n$ и $\pi / m$, где $n, m-$ натуральные числа. Соответствующая плоская структура обозначается через $\widetilde{\omega}_{n, m}$, поверхность, на которой она задана, - через $M_{n, m}$. Поверхность $M_{n, m}$ "склеена" из правильных $n$ - и $m$-угольников с равной стороной таким образом, что каждая сторона произвольного $n$-угольника отождествляется со стороной некоторого $m$-угольника 
(см. [1]). При этом все вершины правильных многоугольников отождествляются, образуя одну особую точку плоской структуры $\widetilde{\omega}_{n, m}$. Кроме указанной особой точки, есть и другие - центры многоугольников. Все последние имеют кратность 1 , поэтому их можно объявить неособыми точками. В результате получаем новую плоскую структуру, которую обозначим $\omega_{n, m}$. Стороны правильных многоугольников являются кратчайшими седлосвязками плоской структуры $\omega_{n, m}$. Декартовы координаты на поверхности $M_{n, m}$ выберем таким образом, чтобы одна из этих кратчайших седлосвязок была горизонтальной.

На первьй взгляд, различие между плоскими структурами $\omega_{n, m}$ и $\widetilde{\omega}_{n, m}$ чисто формальное. Тем не менее, их стабилизаторы могут сильно различаться (см. п. 3 ).

Пусть $k$ - целое число, a $l$ - целое число или $\infty$, причем оба числа не меньше 2 и хотя бы одно из них строго больше 2 . Через $\Gamma_{k, l}$ обозначим подгруппу в $\mathrm{SL}(2, \mathbb{R})$, порожденную элементами

$$
\sigma_{k}=\left(\begin{array}{cc}
\cos \frac{\pi}{k} & -\sin \frac{\pi}{k} \\
\sin \frac{\pi}{k} & \cos \frac{\pi}{k}
\end{array}\right) \quad \text { и } \quad \tau_{k, l}=\left(\begin{array}{cc}
1 & 2 L_{k, l} \\
0 & 1
\end{array}\right)
$$

где

$$
L_{k, l}=\frac{\cos \frac{\pi}{k}+\cos \frac{\pi}{l}}{\sin \frac{\pi}{k}}, \quad L_{k, \infty}=\frac{\cos \frac{\pi}{k}+1}{\sin \frac{\pi}{k}}=\operatorname{ctg} \frac{\pi}{2 k} .
$$

Группа $\Gamma_{k, l}$ является решеткой (см. [1]). Она имеет одну параболическую вершину, если $l$ конечно, и две вершины при $l=\infty$.

ПРЕДЛОЖЕНИЕ 1.2. Справедливы равенства:

1) $\Gamma\left(\omega_{n, n}\right)=\Gamma\left(\omega_{2, n}\right)=\Gamma_{n, 2}$ при нечетном $n$;

2) $\Gamma\left(\omega_{n, n}\right)=\Gamma\left(\omega_{2, n}\right)=\Gamma_{n / 2, \infty}$ при четном $n \geqslant 6$;

3) $\Gamma\left(\omega_{n, 2 n}\right)=\Gamma_{n, 3}$ при любом $n$.

ДокАЗАТЕЛЬСТВо. То, что каждый из перечисленных стабилизаторов содержит соответствующую решетку, показано в [1, теорема 4.4]. Для доказательства утверждения о равенстве соответствующих груп воспользуемся предложением 1.1. В качестве $\bar{v}_{1}$ во всех случаях выбираем горизонтальное направление. Решетка $\Gamma_{n / 2, \infty}$ имеет две параболические вершины, поэтому для плоских структур $\omega_{n, n}$ и $\omega_{2, n}(n-$ четное) выберем направление $\bar{v}_{2}$ под углом $\pi / n$ к горизонтальному. При этом для плоских структур вида $\omega_{2, n}$, где $n$ делится на 4 , в $r$-последовательности, соответствующей направлению $\bar{v}_{2}$, все члены равны, а в $r$-последовательности, соответствующей направлению $\bar{v}_{1}$, один из членов вдвое меньше остальных, если $n \geqslant 8$. Что касается плоских структур $\omega_{n, n}$ $\left(n-\right.$ четное) и $\omega_{2, n}(n-$ четное, не делящееся на 4$)$, то для них $r$-последовательности, соответствующие направлениям $\bar{v}_{1}$ и $\bar{v}_{2}$, имеют разную длину (а именно, первая последовательность на единицу короче второй). Тем самым, проверено одно из условий предложения 1.1 .

Проверим теперь вьполнение условий i) - iv). Условие i) во всех рассматриваемых случаях было установлено в [1], а именно, там показано, что в качестве оператора $b$ можно взять оператор $\tau_{n, m}$ - одну из порождающих соответствующей решетки $\Gamma=\Gamma_{n, m}$. Далее, положим $l$ равным длине кратчайшей седлосвязки. Тогда $\omega_{2, n}(n-$ любое) имеет одну седлосвязку с разверткой $l \bar{v}_{1}$, a $\omega_{n, n}\left(n-\right.$ четное) и $\omega_{n, 2 n}-$ две седлосвязки, которые переводятся друг в друга аффинной симметрией с линейной частью \pm 1 . Заметим, что 
$-1 \in \Gamma_{n, m}$. При этом у плоской структуры $\omega_{n, 2 n}$ седлосвязки с разверткой $l \bar{v}_{1}$ ограничивают два неконгруэнтных пучка, у всех остальных - переходят в себя при аффинной симметрии с линейной частью - 1 . Наконец, параллельньй $\bar{v}_{1}$ периодический пучок, компонента границы которого содержит две седлосвязки равной длины, есть только у плоской структуры $\omega_{n, n}$ при $n$, делящемся на 4. Это пучок, содержащий центры правильных $n$-угольников, образуюших поверхность $M_{n, n}$; после его удаления поверхность (без особой точки) распадается на две связные компоненты, каждая из которых содержит по седлосвязке с разверткой $l \bar{v}_{1}$. Итак, условия i)-iv) проверены.

2. Симметричные периодические траектории. Как заметил Стёпин (см., например, [5]), бильярдная траектория в рациональном многоугольнике, запущенная перпендикулярно какой-нибудь стороне, либо периодична, либо является обобщенной диагональю. Соответствующее рассуждение можно перенести с бильярдњых траекторий на траектории (т.е. геодезические) плоских структур.

ОПРЕДЕЛЕнИЕ 2.1. Аффинное отражсение плоской структуры-это аффинная симметрия, линейная часть которой является оператором отражения относительно прямой (не обязательно ортогональньм) и которая имеет хотя бы одну неособую неподвижную точку.

ОПРЕДЕЛЕНИЕ 2.2. Траектория плоской структуры назьвается симметричной, если найдется аффинное отражение, которое переводит вектор, параллельный этой траектории, в противоположньй и оставляет неподвижной хотя бы одну точку траектории.

ПРЕДЛОЖЕНИЕ 2.1. Симметричная траектория плоской структуры либо периодична, либо является седлосвязкой.

ДокАЗАТЕльСтво. Пусть $L$ - симметричная траектория, $\bar{v}$ - ее направляющий вектор, а точка $x \in L$ и аффинное отражение $S$ таковы, что $S x=x$ и $S \bar{v}=-\bar{v}$. Пусть, далее, $\bar{u}$ - вектор, переводящийся отражением $S$ в себя, и $L_{1}$ - траектория, проходящая через точку $x$ параллельно вектору $\bar{u}$. Каждую точку $y \in L_{1}$ отражение $S$ оставляет неподвижной (отсюда, кстати, следует, что замькание $L_{1}$ не имеет внутренних точек, т.е. $L_{1}$ конечна). Траектория $L$ пересекает $L_{1}$ в точке $x$. Если $L$ не седлосвязка, то она пересечет $L_{1}$ еще раз, скажем, в некоторой точке $y$. Обозначим через $[x, y]$ отрезок траектории $L$ от точки $x$ до точки $y$. Так как $S \bar{v}=-\bar{v}$, отрезки $S([x, y])$ и $[x, y]$ являются смежными отрезками траектории $L$. При этом вся $L$ есть объединение этих отрезков, следовательно, она периодична.

Утверждение о бильярдных траекториях, выпущенных перпендикулярно стороне многоугольника, оказьвается частным случаем доказанного. Действительно, пусть $x-$ точка на стороне $a$ рационального многоугольника $Q$ и $L-$ бильярдная траектория, выпушенная из $x$ перпендикулярно стороне $a$. Рассмотрим поверхность $M$ с плоской структурой, в которую вкладьвается многоугольник $Q$. Бильярдная траектория $L$ выпрямляется до траектории $L_{1}$ плоской структуры. Отражение относительно стороны $a$ порождает аффинное (ортогональное) отражение $S$ плоской структуры. Траектория $L_{1}$ очевидньг образом симметрична относительно отражения $S$, и по доказанному она либо периодична, либо является седлосвязкой. Отсюда следует, что бильярдная траектория $L$ либо периодична, либо является обобщенной диагональю.

Для плоских структур со стабилизатором-решеткой, рассмотренных в п. 1, предложение 2.1 допускает обращение. 
ПРЕДЛОЖЕНИЕ 2.2. ДЛя плоских структур вида $\omega_{n, n}, \omega_{2, n}, \omega_{n, 2 n}$ каждая периодическая траектория и каждая седлосвязка является симметричной.

ДокАЗАТЕЛЬСтво. Пусть $S$ - аффинное отражение, а $T$ - аффинная симметрия плоской структуры, и $L$ - траектория, симметричная относительно отражения $S$. Тогда $T S T^{-1}$ - аффинное отражение, относительно которого симметрична траектория $T(L)$. Таким образом, аффинная симметрия переводит симметричную траекторию в симметричную. Между тем, из доказательств предложений 1.1 и 1.2 вытекает, что любая конечная траектория (периодическая или седлосвязка) плоской структуры, из тех, что упомянуты в условии, переводится аффинной симметрией либо в траекторию, параллельную кратчайшей седлосвязке, либо для плоских структур $\omega_{n, n}$ и $\omega_{2, n}$, где $n$ четно, - в траекторию, параллельную или идущую под углом $\pi / n$ к кратчайшей седлосвязке. А все такие траектории симметричны, причем соответствуюшее аффинное отражение можно выбрать ортогональным.

3. Пучки периодических бильярдных траекторий. Пусть $Q$-рациональный многоугольник, $\omega$ - соответствующая ему плоская структура на поверхности $M$. В [1] рассматривались величины $N_{0}(R), N(R), S(R), N^{*}(R), S^{*}(R)$, обозначающие соответственно число седлосвязок $\omega$ длины не более $R$, число и суммарную площадь некратных пучков периодических траекторий длины не более $R$, затем то же число и суммарную площадь - уже с учетом кратных пучков (траектории в кратном пучке суть несколько раз пройденные траектории меньшей длины). По аналогии с этим введем в рассмотрение величины $\widetilde{N}_{0}(R), \widetilde{N}(R), \widetilde{S}(R), \widetilde{N}^{*}(R), \widetilde{S}^{*}(R)$, характеризующие бильярдньй поток в $Q$. Вместо седлосвязок здесь подсчитываются обобщенные диагонали, вместо пучков периодических геодезических - пучки периодических бильярдных траекторий. Площадью некратного пучка периодических траекторий при этом назьваем произведение его длины на ширину (оно больше, чем площадь подмножества $Q$, занимаемого траекториями пучка). Площадь кратного пучка есть по определению площадь соответствующего некратного пучка.

Пусть $k$ - порядок групшы $K$, порожденной линейными частями отражений относительно сторон многоугольника $Q$. Естественная проекция $\pi: M \rightarrow Q$ является $k$-листной, поэтому обобщенная диагональ многоугольника, как правило, является образом $k$ седлосвязок плоской структуры $\omega$. Аналогично, пучок периодических бильярдных траекторий есть образ $k$ периодических пучков траекторий геодезического потока на $M$ (имеющих такую же площадь). Исключение составляют обобщенные диагонали и периодические траектории, чьи звенья направлены вдоль неподвижных векторов отражений из $K$. Такие обобщенные диагонали и периодические пучки (их конечное число) могут иметь $k / 2$ или $k$ прообразов. (Заметим, что периодический пучок, имеющий $k / 2$ прообразов, содержит внутри себя одну траекторию длины вдвое меньшей, нежели длина пучка, - нечетнозвенную периодическую траекторию [5].) Таким образом, с точностью до постоянного слагаемого $N_{0}(R)=k \widetilde{N}_{0}(R), N(R)=k \widetilde{N}(R)$ и т.д. Если стабилизатор $\Gamma(\omega)$ - решетка, то согласно $[1$, теорема 3.13$]$ все величины $\widetilde{N}_{0}(R), \widetilde{N}(R), \widetilde{S}(R)$, $\widetilde{N}^{*}(R), \widetilde{S}^{*}(R)$ имеют при $R \rightarrow \infty$ асимптотику вида $c R^{2}+o\left(R^{2}\right)$, где $c$-положительная постоянная. Если же стабилизатор-решетку имеет плоская структура, отличающаяся от $\omega$ лишь устранимыми особыми точками, то указанную асимптотику, по-прежнему, имеют величины $\widetilde{S}(R)$ и $\widetilde{S}^{*}(R)$ (так как соответствующие плоской структуре величины $S(R)$ и $S^{*}(R)$ не зависят от устранимых особых точек); об остальных этого утверждать нельзя. 
ПРЕДЛОЖЕНИЕ 3.1. Стабилизаторы плоских структур $\widetilde{\omega}_{2, n} u \widetilde{\omega}_{n, n}$ являются решетками при четном $n>2$ и не являются решетками при нечетном $n>3$.

ДокАЗАтЕльство. Пусть $n$ четно. Покажем, что решетка $\Gamma_{n / 2, \infty}-$ стабилизатор плоских структур $\omega_{2, n}$ и $\omega_{n, n}-$ будет стабилизатором и для $\widetilde{\omega}_{2, n}$ и $\widetilde{\omega}_{n, n}$. То, что плоские структуры $\widetilde{\omega}_{2, n}$ и $\widetilde{\omega}_{n, n}$ допускают аффинную симметрию с линейной частью $\sigma_{n / 2}$, следует из их построения. Далее, пусть $\varphi$ - аффинная симметрия плоской структуры $\omega_{2, n}$ (или $\left.\omega_{n, n}\right)$ с линейной частью $\tau_{n / 2, \infty}$, оставляющая неподвижными горизонтальные седлосвязки. Достаточно показать, что она также является аффинной симметрией структуры $\widetilde{\omega}_{2, n}\left(\right.$ соответственно $\left.\widetilde{\omega}_{n, n}\right)$, т.е. переводит друг в друга особые точки последней. Плоская структура $\widetilde{\omega}_{n, n}$ отличается от $\omega_{n, n}$ двумя особыми точками - центрами $n$-угольников, составляющих поверхность $M_{n, n}$. Если $n$ не делится на 4 , они лежат на горизонтальных седлосвязках $\omega_{n, n}$ и $\varphi$ оставляет их на месте. Если же $n$ делится на 4 , они принадлежат траектории, делящей пополам один из горизонтальных пучков траекторий $\omega_{n, n}$; расстояние между точками равно половине длины пучка, поэтому $\varphi$ меняет их местами. Точно так же особые точки $\widetilde{\omega}_{2, n}$, не лежащие на горизонтальных седлосвязках плоской структуры $\omega_{2, n}$, размещаются по две на траекториях, делящих пополам пучки горизонтальных траекторий $\omega_{2, n}$; расстояние между точками в паре равно половине длины траектории, и симметрия $\varphi$ меняет местами точки в каждой паре.

При нечетном $n$ особые точки $\widetilde{\omega}_{n, n}$ - центры правильных $n$-угольников, составляющих $M_{n, n},-$ расщепляют горизонтальньй пучок траекторий плоской структуры $\omega_{n, n}=\omega_{2, n}$, которому они оба принадлежат, на три части, ширины которых равны $d \sin (\pi /(2 n)), d(\sin (3 \pi /(2 n)-\sin (\pi /(2 n)))$ и $d \sin (\pi /(2 n))$, где $d-$ расстояние от центра правильного $n$-угольника до его вершины. Отношение первых двух из этих величин равно

$$
\frac{\sin \frac{3 \pi}{2 n}-\sin \frac{\pi}{2 n}}{\sin \frac{\pi}{2 n}}=\frac{2 \sin \frac{\pi}{2 n} \cos \frac{\pi}{n}}{\sin \frac{\pi}{2 n}}=2 \cos \frac{\pi}{n},
$$

что иррационально при $n>3$. Особые точки плоской структуры $\widetilde{\omega}_{2, n}$, отличные от особых точек $\widetilde{\omega}_{n, n}$, делят пополам все пучки горизонтальных траекторий плоской структуры $\omega_{n, n}$, поэтому для $\widetilde{\omega}_{2, n}$, равно как и для $\widetilde{\omega}_{n, n}$, отношения длины к ширине горизонтальных пучков не являются попарно соизмеримьми. Тем самым, альтернатива Вича не вьполнена, и стабилизаторы $\Gamma\left(\widetilde{\omega}_{2, n}\right)$ и $\Gamma\left(\widetilde{\omega}_{n, n}\right)$ не являются решетками.

СлЕДСТВИЕ 3.1. Для прямоугольного треугольника с острым углом $\pi / n$ и равнобедренного - с углом при основании $\pi / n$, где $n$ - четное число, величины $\widetilde{N}_{0}(R)$, $\widetilde{N}(R), \widetilde{S}(R), \widetilde{N}^{*}(R), \widetilde{S}^{*}(R)$ имеют при $R \rightarrow \infty$ асимптотику вида $c R^{2}+o\left(R^{2}\right)$, где $c$ - полохительная постоянная.

4. $d$-неустойчивые бильярдные траектории. В связи с изучением бифуркаций периодических траекторий бильярдов в многоугольниках Стёпин ввел понятие деформационной устойчивости ( $d$-устойчивости) таких траекторий. В статье [5] был доказан ряд утверждений о деформационной устойчивости или неустойчивости периодических бильярдных траекторий в различных многоугольниках. Ниже доказьвается результат в этом направлении.

ОПРЕДЕЛЕниЕ 4.1. Периодическая бильярдная траектория в многоугольнике $Q$ назьвается $d$-yстойчивой, если для любого $\varepsilon>0$ в каждом многоугольнике, полученном 
из $Q$ достаточно мальм шевелением сторон и углов, найдется периодическая бильярдная траектория с тем же числом звеньев, которая целиком лежит в $\varepsilon$-окрестности исходной траектории.

ЛЕмма 4.1 [5]. Пусть $a_{1}, \ldots, a_{l}$ - стороны многоугольника $Q$ и $W=a_{i_{1}} \cdots a_{i_{m}}-$ ииклическое слово, сопоставляемое периодической бильярдной траектории (т.е. последовательность сторон, проходимых точкой, движущейся вдоль траектории). Если т четно, то эта траектория d-устойчива в том и только том случае, когда альтернированная сумма $+a_{i_{1}}-a_{i_{2}}+\cdots-a_{i_{m}}$ равна 0 (как әлемент свободного $\mathbb{Z}$-модуля с базисом $\left.a_{1}, \ldots, a_{l}\right)$.

ЛЕмма 4.2. Пусть оба кониа обобщенной диагонали, ограничивающей пучок периодических бильярдных траекторий в многоугольнике, лежсат в вершинах углов величины вида $\pi /(2 n), n \in \mathbb{N}$. Тогда все траектории в этом пучке d-неустойчивы.

ДоКАЗАТЕЛЬСтво. Обозначим каким-то образом стороны многоугольника. Пусть $L$ - рассматриваемая обобщенная диагональ, $A, B$ - ее начало и конец соответственно, а $x_{1}, x_{2}, \ldots, x_{m}$ - стороны, последовательно посещаемые точкой, движущейся по $L$ в направлении от $A$ к $B$. Пусть углы многоугольника при вершинах $A$ и $B$ равны соответственно $\pi /(2 n)$ и $\pi /(2 l)$. Опишем, как расположена в многоугольнике периодическая бильярдная траектория из пучка, ограниченного обобщенной диагональю $L$. Начав движение из точки вблизи вершины $A$, бильярдньй шар движется параллельно обобщенной диагонали $L$ до ее конца, затем он $2 l$ раз поочередно отражается относительно сторон $c$ и $d$ многоугольника, вьходящих из вершины $B$. После этого шар возврашается обратно, двигаясь опять-таки параллельно $L$, но уже с другой стороны от нее. В конце пути он $2 n$ раз отражается относительно сторон $a$ и $b$, выходящих из вершины $A$, после чего возвращается в исходное положение. Таким образом, периодической траектории соответствует слово

$$
W=x_{1} x_{2} \cdots x_{m}(c d)^{l} x_{m} \cdots x_{2} x_{1}(a b)^{n}
$$

(здесь возведениеподслова в $i$-ю степень обозначает его $i$-кратное повторение). Альтернированная сумма букв слова $W$ равна: $S=(-1)^{m} l(c-d)+n(a-b)$. Если вершины $A$ и $B$ не совпадают, то $S \neq 0$, так как в этом случае среди сторон $a, b, c, d$ не менее трех различных. Если же $A=B$, то заметим следующее. Первое звено периодической траектории и ее $(m+1)$-е звено, параллельные соответственно первому и $(m+1)$-му (т.е. последнему) звену $L$, расположены слева или справа от соответствующих звеньев $L$ (левая и правая сторона определены первоначальной ориентацией $L$ ). При этом оба звена траектории расположены по одну сторону (оба слева или оба справа) от звеньев обобщенной диагонали, если $m$ четно, и по разные стороны, если $m$ нечетно. Так как начало первого звена бильярдной траектории находится на стороне $b$, конец $(m+1)$-го звена - на стороне $c$, первое звено $L$ выходит из вершины $A$, a $(m+1)$-е входит в нее, мы получаем, что $a=c, b=d$ при четном $m$ и $a=d, b=c$ при нечетном. В любом случае, $(-1)^{m}(c-d)=a-b$ и $S=2 n(a-b) \neq 0$.

Итак, $S \neq 0$, и согласно лемме 4.1 периодическая бильярдная траектория в пучке, ограничиваемом обобщенной диагональю $L, d$-неустойчива.

ТЕОрема 4.1. В прямоугольном треугольнике с острым углом $\pi /(2 n), n \in \mathbb{N}$, все периодические бильярдные траектории $d$-неустойчивы. 
ДокАЗАТЕЛЬСтво. Напомним, что поверхность с плоской структурой, соответствующая прямоугольному треугольнику с острым углом $\pi /(2 n), n \in \mathbb{N}$, получается из правильного $2 n$-угольника отождествлением его противоположных сторон. Вершинам треугольника соответствуют особые точки плоской структуры. При этом вершинам углов $\pi / 2$ и $\pi /(2 n)$ соответствуют $n+1$ особых точек кратности 1 (центр $2 n$-угольника и середины его сторон), а третьей вершине треугольника - еще одна особая точка $p$, кратность которой больше 1 при $n>3$. Из предложений 1.2 и 3.1 (и их доказательств) следует, что любой пучок периодических геодезических рассматриваемой плоской структуры некоторой аффинной симметрией $\varphi$ переводится в пучок, параллельный стороне или диагонали $2 n$-угольника. Каждьй из последних, как легко видеть, имеет на границе седлосвязку, ни один конец которой не совпадает с $p$. Заметим теперь, что при $n>3$ симметрия $\varphi$ переводит точку $p$ в себя (так как в этом случае $p$ - единственная особая точка, кратность которой больше 1), а при $n=2$ или 3 ее можно выбрать так, чтобы точка $p$ переходила в себя. Следовательно, любой пучок периодических геодезических имеет на гранище подобную седлосвязку. Отсюда получаем, что каждый пучок периодических бильярдных траекторий в исходном треугольнике имеет на границе обобщенную диагональ, оба конца которой находятся в вершинах углов величины $\pi / 2$ или $\pi /(2 n)$. Ввиду леммы 4.2 это завершает доказательство.

В статье [5] другим методом было доказано отсутствие $d$-устойчивых периодических бильярдных траекторий в трех многоугольниках: в прямоугольнике и в прямоугольных треугольниках с острым углом $\pi / 4$ или $\pi / 6$. Как видим, для первых двух многоугольников это утверждение следует из леммы 4.2 , кроме того, для треугольников оно оказьвается частньм случаем теоремы 4.1 .

\section{СПИСОК ЦИТИРОВАННОЙ ЛИТЕРАТУРЫ}

[1] Воробец Я. Б. Плоские структуры и бильяды в рациональных многоугольниках: альтернатива Вича // УМН. 1996. Т. 51. № 5. С. 3-42.

[2] Veech W. A. Teichmüller curves in moduli space, Eisenstein series and an application to triangular billiards // Invent. Math. 1989. V. 97. № 4. P. 553-583.

[3] Воробец Я. Б. Плоские структуры и бильярды в рациональных многоугольниках // УМН. 1996. Т. 51. № 1. C. 145-146.

[4] Бердон А. Геометрия дискретных групп. М.: Наука, 1986.

[5] Воробец Я. Б., Гальперин Г. А., Стёпин А. М. Периодические бильярдные траектории в многоугольниках: механизмы рождения // УМН. 1992. Т. 47. № 3. С. 9-74.

Московский государственный университет

Поступило

им. М.В. Ломоносова

18.02 .97

E-mail : vorobets@nw.math.msu.su 